\title{
MEASURE THEORETICAL APPROACH TO RECURRENT PROPERTIES FOR QUANTUM DYNAMICS
}

\author{
YOSHIKI OTOBE AND ITARU SASAKI
}

\begin{abstract}
Poincaré's recurrence theorem which states that every Hamiltonian dynamics enclosed in a finite volume returns to its initial position as close as one wishes is a mathematical basis of the statistical mechanics. It is Liouville's theorem that guarantees the dynamics preserves the volume on the state space. A quantum version of Poincaré's theorem was obtained in the middle of 20th century without any volume structures of the state space (Hilbert space). One of our aims in the present paper is to establish such properties of quantum dynamics from an analogue of Liouville's theorem, namely, we will construct a natural probability measure on the Hilbert space from a Hamiltonian defined on the space. Then we will show that the measure is invariant under the corresponding Schrödinger flow. We moreover show that the dynamics naturally causes an infinite dimensional Weyl transformation. It also enables us to discuss ergodic properties of such dynamics.
\end{abstract}

\section{INTRODUCTION}

It is believed that a mathematical basis of statistical mechanics is an ergodic property, and a basis of the ergodic theory is Poincaré's recurrence theorem. A classical version of Poincaré's recurrence theorem is stated as follows: Let $X$ be a complete separable metric space with a Borel $\sigma$-field $\mathscr{B}$ and a probability measure $\mu$. If $T: X \rightarrow X$ preserves $\mu$, we have $\mu(\{x \notin \omega(x)\})=0$, where the omega-limit set $\omega(x)$ of $x$ is the set of $y \in X$ such that $\liminf _{n \rightarrow \infty} d\left(T^{n} x, y\right)=0$. If we combine it with Liouville's theorem which states that every Hamiltonian dynamics preserves the Lebesgue measure, we can assert that every Hamiltonian dynamics enforced in a finite volume has a recurrence property.

Since Poincaré's recurrence theorem holds for any measure preserving transformation, we can say that the principal grounds of the recurrence property for classical Hamiltonian dynamics are the Liouville property and the finite volume enforcement. To our knowledge, a quantum version of the recurrence theorem was first

Date: September 28, 2011.

1991 Mathematics Subject Classification. 28D10,58J51.

Key words and phrases. quantum dynamics, Poincaré's recurrence theorem.

Y. O.'s work was supported by KAKENHI 19740047, 22740086, and 22244007.

I. S.'s work was partly supported by KAKENHI Y22740087, and was performed through the Program for Dissemination of Tenure-Track System funded by the Ministry of Education and Science, Japan. 
established by Bocchieri and Loinger [2]. They showed that if the quantum Hamiltonian has purely point spectrum, a recurrence theorem holds for the quantum dynamics. They used some properties of almost-periodic functions to show the theorem; without the Liouville property or the finite volume enforcement. After that Shulman [17] gave another proof of the theorem using the classical Poincaré's recurrence theorem, still without a concept of a probability measure on the state space.

In Section 2, we shall note a result that the recurrence theorem never holds for the initial state belonging to the absolutely continuous subspace of the Hamiltonian. We also give a counterexample for the recurrence property if the initial state belongs to the singular continuous spectrum. We do not know whether the non-recurrence holds for all singular continuous states. We, however, want to emphasize here that this example shows a crucial difference from the scattering theory. In the scattering theory, it is known by [19] that, if a particle spends finite time in every finite region in space, then the state is absolutely continuous. And if the initial state is singular continuous, there exists at least one finite region in space that the particle spends infinite time of sojourn there. Our example shows, however, from the recurrence point of view, nonrecurrent property could be shared by absolutely and singular continuous spectra.

We will then introduce an analogue of the Liouville property for the quantum dynamics in Section 3. Recall that, in a usual setting for analysis on infinite dimensional spaces, Gaussian measures will play a similar role to the Lebesgue measure on finite dimensional spaces. In our context, Gaussian measures in quantum mechanics play a same role as the Lebesgue measure in classical mechanics. We, however, emphasize here that the Gaussian measures in the present paper will be constructed from our Hamiltonian.

The finite volume enforcement reflects on the existence of the probability measure on the Hilbert space; recall that existence of the finite (or probability) Lebesgue measure is equivalent to it in classical cases. We believe that this approach makes a natural way to discuss ergodic properties of the dynamics. Needless to say that the quantum dynamics never has an ergodic property since all balls centered at origin are invariant under the Schrödinger (unitary) flow. In Section 4, we shall disintegrate the measure and represent the dynamics as a Weyl transformation on an infinite dimensional torus. Then we will recover a "Lebesgue measure" on such infinite dimensional spaces and give a simple condition that the dynamics is ergodic with respect to that measure. We expect that it also shows the reason why taking Gaussian measures on the Hilbert space is natural in our setting. Although the Gaussian measures constructed from the Hamiltonian are not uniquely determined, the dynamics on the infinite dimensional torus is the same.

To close the introductory section, we want to give comments for related studies. A computer experiments of the quantum recurrence is examined by Hogg and 
Huberman [7]. H-theorem for such systems was treated by Percival [14]. The recurrence properties and the Liouville properties are also studied by Duvenhage in a context of $C^{*}$-algebra [6]. There are several studies in $C^{*}$-algebras, see e.g., [15].

On the other hand, mathematically speaking, our dynamics are a simple example of dynamics of linear operators which has been a very active field in these years, see e.g., [1]. It is also worthy to point out that, since our measures are invariant under the Schrödinger flow, we carry out construction of invariant measures for certain linear partial differential equations of Schrödinger type (see Corollary 6). Our result may be interesting from the point of view to construct invariant measures for the deterministic dynamics governed by partial differential equations. We only indicate $[5,4]$ for this direction.

Both authors would like to express our deeply gratitude to kind hospitality of the Isaac Newton Institute for Mathematical Sciences in Cambridge. A part of this research was executed during our stay at the institute. We would also like to thank Zdzisław Brzeźniak for his comments and suggestions at an early stage of the study. His insight and discussions enabled us to refine the present paper. Finally we are grateful to anonymous referees, who informed us about the Rajchman measures and related studies, and suggested several instructive improvements of the present paper.

\section{Functional Analytic Approach}

This section treats the recurrence theorem without any measures. Throughout the paper, we denote by $\mathscr{H}$ the separable complex Hilbert space on which a (fixed, single) self-adjoint operator $H$ is defined. We decompose $\mathscr{H}$ into $\mathscr{H}_{\mathrm{pp}} \oplus \mathscr{H}_{\mathrm{sc}} \oplus \mathscr{H}_{\mathrm{ac}}$; pure point, singular continuous, absolutely continuous spectrum, respectively, by $H$.

Definition 1. We say that a vector $\Psi \in \mathscr{H}$ is recurrent if $\lim _{\inf _{t \rightarrow \infty}} \| e^{-\sqrt{-1} t H} \Psi-$ $\Psi \|_{\mathscr{H}}=0$.

One can easily observe the following result.

Theorem 1. (1) Every $\Psi \in \mathscr{H}_{\mathrm{pp}} \backslash\{0\}$ is recurrent.

(2) No $\Psi \in \mathscr{H}_{\mathrm{ac}} \backslash\{0\}$ is recurrent.

Proof. The proof of the first part of the theorem can be found in $[2,17]$. We will give a proof for the second part. Let $\Psi \in \mathscr{H}_{\text {ac }} \backslash\{0\}$. We clearly have

$$
\left\|e^{-\sqrt{-1} t H} \Psi-\Psi\right\|_{\mathscr{H}}^{2}=2\|\Psi\|_{\mathscr{H}}^{2}-2 \Re \int_{\mathbb{R}} e^{-\sqrt{-1} t \lambda} d\langle\Psi, E(\lambda) \Psi\rangle,
$$

where $E(\cdot)$ denotes the spectral measure of $H$, namely, a family of projection operators $\{E(\Lambda) ; \Lambda \in \mathscr{B}(\mathbb{R})\}$ is a resolution of the identity on $\mathbb{R}$. The second term converges to zero from the Radon-Nikodym's theorem, the Riemann-Lebesgue's lemma, and the assumption $\Psi \in \mathscr{H}_{\text {ac }}$, which implies the conclusion. 
Therefore the difference between pure point and absolutely continuous spectrum is clear. Next we will consider a case of singular continuous case. We show a counterexample for the recurrence. If the spectral measure is Rajchman, the state is not recurrent ([12]). Our example shows, however, that even if the spectral measure is not Rajchman, there is a case that the state is nonrecurrent. As we already pointed out in Section 1, it might be conjectured that nonrecurrent property could be shared by absolutely and singular continuous spectra, and shows that such recurrence phenomena differ from the scattering theory.

Before showing the example, we give some notations. Let $\varphi:[0,1] \rightarrow[0,1]$ be a Cantor function and $\mu$ be the Borel probability measure on $[0,1]$ whose distribution function is given by $\varphi$, i.e., $\mu((\lambda, \mu))=\varphi(\mu)-\varphi(\lambda),(0 \leq \lambda, \mu \leq 1)$ and

$$
\mu(B)=\inf \{\mu(O) \mid B \subset O, O \text { open }\}
$$

on Borel sets of $[0,1]$. Take $\mathscr{H}_{\mathrm{C}}:=L^{2}([0,1], \mu)$ and $H_{\mathrm{C}}$ as a multiplication operator by $\varphi(\lambda)$. We define a projection operator $[E(\Lambda) \Psi](\lambda):=1_{\Lambda}(\lambda) \Psi(\lambda)$ for $\Psi \in \mathscr{H}_{\mathrm{C}}$ and $\Lambda \in \mathscr{B}([0,1])$. Then we have $H_{\mathrm{C}}=\int_{0}^{1} \lambda d E(\lambda)$. It is clear that $H_{\mathrm{C}}$ has purely singular continuous spectrum, i.e., $\sigma\left(H_{\mathrm{C}}\right)=\sigma_{\mathrm{sc}}\left(H_{\mathrm{C}}\right), \sigma_{\mathrm{ac}}\left(H_{\mathrm{C}}\right)=\emptyset, \sigma_{\text {p.p. }}\left(H_{\mathrm{C}}\right)=\emptyset$.

Proposition 2. All vectors in $\mathscr{H}_{\mathrm{C}} \backslash\{0\}$ are not recurrent in the dynamics of $e^{-\sqrt{-1} t H_{\mathrm{C}}}$.

Proof. Let $\Psi \in \mathscr{H}_{\mathrm{C}} \backslash\{0\}$ be an arbitrary nonzero vector. For any $\varepsilon>0$ there exist $\alpha_{n} \in \mathbb{C}$ and Borel set $B_{n}$ in $[0,1]$ such that

$$
\begin{aligned}
& |\Psi(\lambda)| \geq\left|\Psi_{n}(\lambda)\right| \quad \lambda \in[0,1], \\
& \Psi_{n}(\lambda)=\sum_{n=1}^{N} \alpha_{n} \chi_{B_{n}}(\lambda)
\end{aligned}
$$

and $\left\|\Psi-\Psi_{n}\right\|<\varepsilon$ hold. Then we have

$$
\left\|e^{-\sqrt{-1} t H_{\mathrm{C}}} \Psi-\Psi\right\|^{2} \geq\left\|e^{-\sqrt{-1} t H_{\mathrm{C}}} \Psi_{n}-\Psi_{n}\right\|^{2}=\sum_{n=1}^{N} \alpha_{n}^{2}\left\|e^{-i t H_{\mathrm{C}}} \chi_{B_{n}}-\chi_{B_{n}}\right\|^{2} .
$$

Therefore it is suffices to prove that for any Borel set $B$ with $\chi_{B} \neq 0$, the characteristic function is not recurrent:

$$
\liminf _{t \rightarrow \infty}\left\|e^{-\sqrt{-1} t H_{C}} \chi_{B}-\chi_{B}\right\|^{2}>0 .
$$

By the outer regularity (1) of $\mu_{\mathrm{C}}$, there exists an open set $O$ such that $B \subset O \subset$ $[0,1]$ with $\left\|\chi_{B}-\chi_{O}\right\|<\varepsilon$. Then we have

$$
\left\|e^{-\sqrt{-1} t H_{\mathrm{C}}} \chi_{B}-\chi_{B}\right\| \geq\left\|e^{-\sqrt{-1} t H_{\mathrm{C}}} \chi_{O}-\chi_{O}\right\|-2 \varepsilon .
$$

Since $O$ is a disjoint union of open intervals, it is sufficient to prove that, for each interval $I \subset[0,1]$

$$
\liminf _{t \rightarrow \infty}\left\|e^{-\sqrt{-1} t H_{C}} \chi_{I}-\chi_{I}\right\| \geq A\left\|\chi_{I}\right\|
$$


holds where $A>0$ is a constant independent of $I$. For $N \in \mathbb{N}$, we consider the set

$$
\left\{\sum_{n=1}^{N} \frac{c_{n}}{3^{n}} \mid c_{n} \in\{0,2\}, n=1,2, \ldots, N\right\},
$$

and write this set as $\left\{a_{1}, a_{2}, \cdots, a_{2^{N}} \mid a_{1}<a_{2}<\cdots<a_{2^{N}}\right\}$. We set

$$
C_{k}^{N}:=\left[a_{k}^{N}, a_{k}^{N}+\frac{1}{3^{N}}\right], \quad C_{N}:=\cup_{k=1}^{2^{N}} C_{k}^{N}, \quad k=1,2, \ldots, 2^{N} .
$$

Clearly, the sequence $\left\{C_{N}\right\}_{N}$ is decreasing: $C_{1} \supset C_{2} \supset \cdots C_{3} \supset \cdots$, and the Cantor set is equal to

$$
C=\lim _{N \rightarrow \infty} C_{N}
$$

Next we show that

$$
\liminf _{t \rightarrow \infty}\left\|e^{-\sqrt{-1} t H_{C}} \chi_{C_{k}^{N}}-\chi_{C_{k}^{N}}\right\| \geq A\left\|\chi_{C_{k}^{N}}\right\| .
$$

From the self-similarity of the Cantor function, we have $2 \mu_{\mathrm{C}}(d \lambda / 3)=\mu_{\mathrm{C}}(d \lambda)$ and

$$
\int_{0}^{1 / 3} f(\lambda) \mu_{\mathrm{C}}(d \lambda)=\int_{0}^{1} f\left(\frac{t}{3}\right) \mu_{\mathrm{C}}\left(\frac{d \lambda}{3}\right)=\frac{1}{2} \int_{0}^{1} f\left(\frac{\lambda}{3}\right) \mu_{\mathrm{C}}(d \lambda)
$$

for any measurable function $f$. Hence we have

$$
\begin{aligned}
\left\|e^{-\sqrt{-1} t H_{\mathrm{C}}} \chi_{C_{k}^{N}}-\chi_{C_{k}^{N}}\right\|^{2} & =\frac{1}{2} \int_{0}^{3 / 3^{N}}\left|e^{-\sqrt{-1} t\left(a_{k}^{N}+\lambda / 3\right)}-1\right|^{2} \mu_{\mathrm{C}}(d \lambda) \\
& =\frac{2}{2^{N}}\left(1-\Re e^{\sqrt{-1} t a_{k}^{N}} \int_{0}^{1} e^{-i t \lambda / 3^{N}} \mu_{\mathrm{C}}(d \lambda)\right) \\
& \geq \frac{2}{2^{N}}\left(1-\left|I\left(t / 3^{N}\right)\right|\right),
\end{aligned}
$$

where $I(t)=\int_{0}^{1} e^{-\sqrt{-1} t \lambda} \mu_{\mathrm{C}}(d \lambda)$. Similarly we can compute

$$
I(t)=\frac{1+e^{-2 \sqrt{-1} t / 3}}{2} I(t / 3) \text {. }
$$

So we have

$$
I(t)=\prod_{j=1}^{\infty}\left(\frac{1+e^{-2 \sqrt{-1} t / 3^{j}}}{2}\right)
$$

and $|I(t)|=\prod_{j=1}^{\infty}\left|\cos \left(t / 3^{j}\right)\right|$. For $t \in[\pi, 3 \pi]$, we have $|\cos (t / 3) \cos (t / 9)|<0.6$ and $|I(t)|<0.6$. The equality $|I(t)|=|\cos (t / 3) I(t / 3)|$ implies $|I(t)|<0.6$ for $t \in[3 \pi, 9 \pi]$. By the inductive argument, we have

$$
|I(t)| \leq 0.6 \quad \text { for } \quad t \geq \pi
$$

By using this fact, the right hand side of (8) is bounded by

$$
0.8 / 2^{N}=0.8 \mu_{\mathrm{C}}\left(C_{k}^{N}\right)=0.8\left\|\chi_{C_{k}^{N}}\right\|^{2},
$$


which implies (4) with $A^{2}=0.8$. Let $I$ be an arbitrary open interval in $[0,1]$. Then, there is at most a countable sequence $\left\{d_{n}\right\}_{n} \subset\left\{C_{k}^{N} \mid N \in \mathbb{N}, k=\left\{1, \ldots, 2^{N}\right\}\right\}$ such that $d_{n} \subset I$ and

$$
\begin{array}{r}
\mu_{\mathrm{C}}\left(d_{n} \cap d_{m}\right)=0 \quad n \neq m \\
\left\|\chi_{I}\right\|^{2}=\mu_{\mathrm{C}}(I)=\sum_{n} \mu_{\mathrm{C}}\left(d_{n}\right) .
\end{array}
$$

Therefore we have

$$
\begin{aligned}
\liminf _{t \rightarrow \infty}\left\|e^{-\sqrt{-1} t H_{\mathrm{C}}} \chi_{I}-\chi_{I}\right\|^{2} & =\liminf _{t \rightarrow \infty} \sum_{n}\left\|e^{-\sqrt{-1} t H_{\mathrm{C}}} \chi_{d_{n}}-\chi_{d_{n}}\right\|^{2} \\
& \geq 0.8 \sum_{n}\left\|\chi_{d_{n}}\right\|^{2}=0.8\left\|\chi_{I}\right\|^{2}
\end{aligned}
$$

and hence (3) holds.

Remark 1. It is conjectured that every state is non-recurrent in the dynamics of the Hamiltonian with purely singular continuous spectrum.

Example 2. Here are some examples of Hamiltonians whose spectral type are pure point. See, e.g., [16] for detailed treatments.

(1) Laplacian in a bounded domain: Let $\Omega$ be a bounded domain in $\mathbb{R}^{d} . H=$ $-\Delta$ on $\mathscr{H}=L^{2}(\Omega, d x)$ with Dirichlet boundary condition has compact resolvent. Hence any $\Psi \in \mathscr{H}$ is recurrent under $e^{-\sqrt{-1} t H}$.

(2) Schrödinger operator with a binding potential: Let $V: \mathbb{R}^{d} \rightarrow \mathbb{R}$ be a continuous real valued function with $V(x) \rightarrow \infty$ as $|x| \rightarrow \infty$. Then $H=-\Delta+V(x)$ on $\mathscr{H}=L^{2}\left(\mathbb{R}^{d}, d x\right)$ has purely pure point spectrum.

(3) Free quantum field in a bounded domain: Let $\Omega$ be a bounded domain in $\mathbb{R}^{d}$ and $\Delta$ be a Laplace operator with Dirichlet boundary condition. Then the second quantization $d \Gamma(\sqrt{-\Delta})$ has compact resolvent since $-\Delta$ is strictly positive.

(4) Interacting quantum field in a bounded domain: Let $\mathscr{K}$ be a Hilbert space and put $\mathscr{H}:=\mathscr{K} \otimes \mathcal{F}_{b}\left(L^{2}(\Omega \times\{1,2\})\right)$, where $\Omega$ is a bounded domain in $\mathbb{R}^{d}$. Let $A$ be a self-adjoint operator on $\mathscr{K}$ and has compact resolvent, and $H_{\mathrm{I}}$ be an interaction Hamiltonian acting on $\mathscr{H}$. Assume $H=A \otimes I+I \otimes$ $d \Gamma(\sqrt{-\Delta})+H_{\mathrm{I}}$ is self-adjoint on $D(A \otimes I+I \otimes d \Gamma(\sqrt{-\Delta}))$. Then the closed graph theorem implies that $H$ has compact resolvent, in particular $H$ has purely pure point spectrum.

(5) Anderson model: Schrödinger operator with suitable random potential in one dimension has purely point spectrum [20]. Then all states are recurrent almost surely.

The above argument can be extended for any self-similar measure and a multiplication operator. Therefore, in general, a state belonging to a singular continuous spectrum is not recurrent. 


\section{Invariant Measures For Schrödinger Flows}

In this section, we assume $H: \mathscr{H} \rightarrow \mathscr{H}$ is a self-adjoint operator which has purely point spectrum with finite degrees of degeneracy, i.e., $\sigma(H)=\overline{\sigma_{\text {p.p. }}(H)}, \sigma_{\mathrm{c}}(H)=$ $\emptyset$ and $\operatorname{dim} \operatorname{ker}(H-\lambda)<\infty$ for all $\lambda \in \sigma(H)$, where $\sigma_{\text {p.p. }}(H)$ and $\sigma_{\mathrm{c}}(H)$ denote the pure point spectrum and the continuous spectrum of $H$, respectively. Since $\sigma_{\text {p.p. }}$. is countable, there exists an injection $f: \sigma_{\text {p.p. }}(H) \rightarrow(0, \infty)$ such that $f(H)$ is of trace class. Note that $Q:=f(H)$ is a strictly positive trace class operator on $\mathscr{H}$. Therefore we can take a complete orthonormal system $\left\{e_{j}\right\}$ such that

$$
Q e_{j}=q_{j} e_{j}
$$

for every $q_{j}>0$ and $\sum_{j=1}^{\infty} q_{j}<\infty$. We start with constructing a mean zero Gaussian measure on $\mathscr{H}$ with covariance operator $Q$; see also section 5.1 of [1].

We first define a Gaussian measure $\mu_{q}(d z)$ on $\mathbb{C}$ :

$$
\mu_{q}(d z):=\frac{1}{\pi q} \exp \left\{-\frac{1}{q}|z|^{2}\right\} d z,
$$

where $q>0$. Note that $d z=d x d y$ is a volume (not length) element of $\mathbb{C}$ for $z=x+\sqrt{-1} y, x, y \in \mathbb{R}$. Recall that $\mu_{q}$ is entirely characterized by its characteristic function

$$
\hat{\mu}_{q}(w):=\int_{\mathbb{C}} \exp \{\sqrt{-1} \Re\langle z, w\rangle\} \mu(d z)=\exp \left\{-\frac{q}{4}|w|^{2}\right\}
$$

for $w \in \mathbb{C}$.

Remark 3. If we use a polar coordinate $z=r e^{\sqrt{-1} \theta}, r \geq 0, \theta \in[0,2 \pi)$, for $z \in \mathbb{C}$, we can rewrite $\mu_{q}$ as follows:

$$
\mu_{q}(d r d \theta)=\frac{r}{\pi q} \exp \left\{-\frac{1}{q} r^{2}\right\} d r d \theta
$$

Let $\left\{n_{1}, n_{2}, \ldots, n_{j}\right\}$ be any finite sequence of positive integers. We can define a Gaussian measure $\mu_{\left\{n_{1}, n_{2}, \ldots, n_{j}\right\}}$ on $\mathbb{C}^{j}$ as

$$
\mu_{\left\{n_{1}, n_{2}, \ldots, n_{j}\right\}}\left(d z_{n_{1}} d z_{n_{2}} \ldots d z_{n_{j}}\right):=\mu_{q_{n_{1}}}\left(d z_{n_{1}}\right) \mu_{q_{n_{2}}}\left(d z_{n_{2}}\right) \ldots \mu_{q_{n_{j}}}\left(d z_{n_{j}}\right),
$$

whose characteristic function is given by

$$
\hat{\mu}_{\left\{n_{1}, n_{2}, \ldots, n_{j}\right\}}(w)=\exp \left\{-\frac{1}{4}\left\langle Q_{n_{1}, \ldots n_{j}} w, w\right\rangle_{\mathbb{C}^{j}}\right\}
$$

for $w \in \mathbb{C}^{j}$, where $Q_{n_{1}, \ldots n_{j}}$ is a restricted matrix of $Q$ on $\operatorname{Span}\left\{e_{n_{1}}, e_{n_{2}}, \ldots, e_{n_{j}}\right\}$.

Therefore, by virtue of Kolmogorov's extension theorem (see [11]), there exists a probability measure $\mu_{Q}$ on $\mathbb{C}^{\infty}$ whose characteristic functional is given by

$$
\hat{\mu}_{Q}(w)=\exp \left\{-\frac{1}{4}\langle Q w, w\rangle_{\mathbb{C}^{\infty}}\right\}
$$


that we call mean zero Gaussian measure on $\mathbb{C}^{\infty}$ with covariance operator $Q$. Moreover, it is not difficult to show that $\mu_{Q}$ is actually supported on

$$
\ell^{2}(\mathbb{C}):=\left\{\left(z_{j}\right)_{j=1}^{\infty} ; \sum_{j=1}^{\infty}\left|z_{j}\right|^{2}<\infty\right\},
$$

and it can be regarded as a Borel probability measure on $\ell^{2}(\mathbb{C})$. Hence, by a standard identification procedure, $\mu_{Q}$ can be regarded as a mean zero Gaussian measure on $\mathscr{H}$ with covariance operator $Q$.

Remark 4. If we introduce another Hilbert space $\mathscr{K}:=Q^{1 / 2}(\mathscr{H})$ with inner product $\langle f, g\rangle_{\mathscr{K}}:=\left\langle Q^{-1 / 2} f, Q^{-1 / 2} g\right\rangle_{\mathscr{H}}$, and if we denote by $\mathscr{H}^{*}$ the dual space of $\mathscr{H}$ under an identification with $\mathscr{K} \simeq \mathscr{K}^{*}$ (Riesz identification), we have $\mathscr{H}^{*} \simeq Q(\mathscr{H}) \subset \mathscr{K}$ and $\langle w, z\rangle_{\mathscr{H}^{*}, \mathscr{H}}=\left\langle Q^{-1} w, z\right\rangle_{\mathscr{H}}$. With these notions, the characteristic functional of $\mu_{Q}$ is given by

$$
\hat{\mu}_{Q}(w)=\int_{\mathscr{H}} \exp \left\{\sqrt{-1} \Re\langle w, z\rangle_{\mathscr{H}^{*}, \mathscr{H}}\right\} \mu_{Q}(d z)=\exp \left\{-\frac{1}{4}\|w\|_{\mathscr{K}}^{2}\right\}
$$

for every $w \in \mathscr{H}^{*}$ and hence $w \in \mathscr{K}$. $\mathscr{K}$ is referred to as a reproducing kernel Hilbert space of $\mu_{Q}$. In the case that $Q=(-\Delta)^{-1}$ on $L^{2}([0, T])$ assuming $Q$ satisfies our assumptions, it can be shown that $\langle w, z\rangle_{\mathscr{K}}$ is a Wiener integral $\int_{0}^{T} w^{\prime}(t) d \bar{z}(t)$.

Remark 5. To our knowledge, Gaussian measures on complex spaces are first studied by Itô [8] followed by first attempts for mathematically rigorous treatment of the Feynman path integrals $[9,10]$. Although our definition may look slightly different from those, it is not difficult to see that our measure determines same Gaussian system in the sense of Itô.

We say a measurable map $T:(X, \mathscr{B}) \rightarrow(X, \mathscr{B})$ preserves a measure $\mu$ on $(X, \mathscr{B})$ if $\mu\left(T^{-1} A\right)=\mu(A)$ for every $A \in \mathscr{B}$.

Lemma 3. Let $\mu_{Q}$ be a mean zero Gaussian measure with covariance operator $Q$ on $\mathscr{H}$. A bounded linear operator $T: \mathscr{H} \rightarrow \mathscr{H}$ preserves $\mu_{Q}$ if and only if $T Q T^{\star}=Q$, where $T^{\star}: \mathscr{H} \rightarrow \mathscr{H}$ is a Hilbert adjoint of $T$.

Proof. It is enough to show that $T \circ \mu_{Q}=\mu_{T Q T^{\star}}$. To do it, it is necessary and sufficient that both characteristic functionals agree. We then have

$$
\begin{aligned}
\widehat{T \circ \mu_{Q}}(h) & =\int_{\mathscr{H}} \exp \left\{\sqrt{-1} \Re\langle h, z\rangle_{\mathscr{H}}\right\}\left(T \circ \mu_{Q}\right)(d z) \\
= & \int_{\mathscr{H}} \exp \left\{\sqrt{-1} \Re\langle h, T z\rangle_{\mathscr{H}}\right\} \mu_{Q}(d z)=\int_{\mathscr{H}} \exp \left\{\sqrt{-1} \Re\left\langle T^{\star} h, z\right\rangle_{\mathscr{H}}\right\} \mu_{Q}(d z) \\
& =\exp \left\{-\frac{1}{4}\left\langle Q T^{\star} h, T^{\star} h\right\rangle_{\mathscr{H}}\right\}=\exp \left\{-\frac{1}{4}\left\langle T Q T^{\star} h, h\right\rangle_{\mathscr{H}}\right\}=\widehat{\mu_{T Q T^{\star}}}(h) .
\end{aligned}
$$

Therefore we have the conclusion. 
Corollary 4. Let $U: \mathscr{H} \rightarrow \mathscr{H}$ be unitary. Then $U$ preserves the measure $\mu_{Q}$ if and only if $U$ commutes with $Q$, that is, $Q U=U Q$.

Proof. Since $U^{\star}=U^{-1}$ on $\mathscr{H}$, it is immediate from Lemma 3.

We recall here that our Gaussian measure $\mu_{Q}$ is entirely determined from the quantum Hamiltonian $H$, that is, we start with a Hilbert space $\mathscr{H}$ and $H$, then we have constructed $\mu_{Q}$ on $\mathscr{H}$ from $H$. Therefore we have the following proposition:

Proposition 5. Assume that $H: \mathscr{H} \rightarrow \mathscr{H}$ is a self-adjoint operator with purely point spectrum with finite degrees of degeneracy. Let $Q:=f(H)$ be a strictly positive trace class operator, where $f: \sigma_{\text {p.p. }}(H) \rightarrow(0, \infty)$ be an injective map. Then the Schrödinger flow $U_{t}:=e^{-\sqrt{-1} t H}$ preserves a mean zero Gaussian measure $\mu_{Q}$ on $\mathscr{H}$ with a covariance operator $Q$ for every $t>0$.

Proof. Since the unitary operator $U_{t}$ commutes with $Q$ for every $t>0$, Corollary 4 asserts that $\mu_{Q}$ is invariant under $U_{t}$.

Corollary 6. Let us consider the following linear partial differential equation of Schrödinger type:

$$
\sqrt{-1} \hbar \frac{\partial \Psi(t)}{\partial t}=H \Psi(t)
$$

If the law of initial state $\Psi(0)$ is $\mu_{Q}$ on $L^{2}\left(\mathbb{R}^{d}, d x\right)$, then the law of $\Psi(t)$ is also $\mu_{Q}$ for every $t>0$.

Remark 6. If we define an operator

$$
\mathscr{U}_{t} F(w):=F\left(U_{t}^{-1} w\right)
$$

for every $w \in \mathscr{H}$, it is easy to see that $\mathscr{U}_{t}$ defines a unitary flow on $L^{2}\left(\mathscr{H}, \mu_{Q}\right)$. To see it, since $\mu_{Q}$ is invariant under $U_{t}$,

$$
\begin{aligned}
\left\langle\mathscr{U}_{t} F, \mathscr{U}_{t} G\right\rangle_{L^{2}\left(\mathscr{H}, \mu_{Q}\right)}= & \int_{\mathscr{H}} F\left(U_{t}^{-1} w\right) \bar{G}\left(U_{t}^{-1} w\right) \mu_{Q}(d w) \\
& =\int_{\mathscr{H}} F\left(U_{t}^{-1} w\right) \bar{G}\left(U_{t}^{-1} w\right)\left(U_{t} \circ \mu_{Q}\right)(d w)=\langle F, G\rangle_{L^{2}\left(\mathscr{H}, \mu_{Q}\right)}
\end{aligned}
$$

for every $F, G \in L^{2}\left(\mathscr{H}, \mu_{Q}\right)$. It is well known that $L^{2}\left(\mathscr{H}, \mu_{Q}\right)=\bigoplus_{n=0}^{\infty} \mathscr{H}^{n}$ by some Hilbert spaces $\mathscr{H}^{n}$ which are defined through Wiener integrals (see e.g., $[8,18,21]$ ) and is isomorphic to a Bosonic Fock space. This means that every Hamiltonian automatically defines a quantum time evolution on a Fock space.

\section{Quantum Liouville's Theorem And ERgodicity}

The classical version of Liouville's theorem states that every classical Hamiltonian dynamics preserves the Lebesgue measure on $\mathbb{R}^{d}$. In the previous section, we constructed an invariant Gaussian measure $\mu_{Q}$ from the quantum Hamiltonian $H$ 
on $\mathscr{H}$. It may give a sense of incongruity if we call it a quantum version of Liouville's theorem; the measure is never universal in the sense that it heavily depends on the Hamiltonian itself, it is not unique (since it depends on the choice of $f$ ), and it is not a uniform measure. As we have already pointed out in Section1, one advantage to treat recurrence properties from a metrical point of view is that it enables us to discuss ergodic properties. Here we say a measure preserving transformation $T:(X, \mathscr{B}, \mu) \rightarrow(X, \mathscr{B}, \mu)$ is ergodic if $\mu\left(T^{-1} A \backslash A\right)=0(A$ is invariant $)$ implies $\mu(A)=0$ or 1 for every $A \in \mathscr{B}$. Unfortunately, our Gaussian measure is never ergodic.

Example 7 ([3]). Let $U: \mathscr{H} \rightarrow \mathscr{H}$ be a unitary transformation on a Hilbert space $\mathscr{H}$ and $\mu$ a Gaussian probability measure on $\mathscr{H}$ with full support. Define $R_{n}:=\left\{z \in \mathscr{H} ; n-1 \leq\|z\|_{\mathscr{H}}<n\right\}$. Then we have $R_{n}$ is invariant under $U$ and $\mathscr{H}=\bigcup_{n=1}^{\infty} R_{n}$. Therefore if $U$ is ergodic, there must be exactly one $n$ such that $\mu\left(R_{n}\right)=1$ and $\mu\left(R_{m}\right)=0$ for $m \neq n$. Since $\mu$ is Gaussian, its restriction to every finite dimensional subspace is also Gaussian, which contradicts the assumption that $\mu$ has full support.

This example implies that, since our dynamics is unitary, we have to restrict our consideration on a sphere. Moreover if we expand $\Psi=\sum \Psi_{j} e_{j}$, where $\left(e_{j}\right)$ is an orthonormal base associated with $H$, the unitary evolution does not change $\left(\Psi_{j}\right)$.

Recall that $\mu_{Q}$ was first constructed on $\ell^{2}(\mathbb{C})$ as an extension of measures on $\mathbb{C}^{n}$. As we already pointed out at Remark 3 , we can disintegrate $\mu_{Q}$ into a product space of position and momentum: Let

$$
\ell_{+}^{2}:=\left\{\left(r_{j}\right)_{j=1}^{\infty} ; r_{j} \geq 0, \sum_{j=1}^{\infty} r_{j}^{2}<\infty\right\} .
$$

Then there exists a canonical map $\ell^{2}(\mathbb{C}) \rightarrow \ell_{+}^{2} \times \mathbb{T}^{\infty}$, where $\mathbb{T}^{\infty}:=\left\{\left(\theta_{j}\right)_{j=1}^{\infty} ; \theta_{j} \in\right.$ $[0,2 \pi)\}$, by $\left(z_{j}\right)_{j=1}^{\infty} \mapsto\left(\left(\left|z_{j}\right|\right)_{j=1}^{\infty},\left(\arg z_{j}\right)_{j=1}^{\infty}\right)$. Therefore, from the construction of $\mu_{Q}$, we can define two probability measures $\rho(d r)$ and $\lambda(d \theta)$ on $\ell_{+}^{2}$ and $\mathbb{T}^{\infty}$, respectively, formally written by

$$
\begin{gathered}
\rho(d r)=\prod_{j=1}^{\infty} \frac{2 r_{j}}{q_{j}} \exp \left\{-\frac{1}{q_{j}} r_{j}^{2}\right\} d r_{j}, \\
\lambda(d \theta)=\prod_{j=1}^{\infty} \frac{1}{2 \pi} d \theta_{j},
\end{gathered}
$$

for $r=\left(r_{1}, r_{2}, \ldots\right) \in \ell_{+}^{2}$ and $\theta=\left(\theta_{1}, \theta_{2}, \ldots\right) \in \mathbb{T}^{\infty}$.

Let us consider, for the sake of simplicity, a unitary operator $U: \mathscr{H} \rightarrow \mathscr{H}$ defined by $U \Psi=e^{-\sqrt{-1} H} \Psi$. Then $U$ is naturally defined as an operator on $\ell_{+}^{2} \times \mathbb{T}^{\infty}$ as follows: Let $\Psi=\sum_{j=1}^{\infty} \Psi_{j} e_{j}$, where $\left(e_{j}\right)$ satisfies $Q e_{j}=q_{j} e_{j}$, or by the definition 
$H e_{j}=f^{-1}\left(q_{j}\right) e_{j} \equiv h_{j} e_{j}$. Then we have

$$
U \Psi=U \sum_{j=1}^{\infty} \Psi_{j} e_{j}=\sum_{j=1}^{\infty} e^{-\sqrt{-1} h_{j}} \Psi_{j} e_{j} .
$$

Let $\Pi: \mathscr{H} \rightarrow \ell_{+}^{2} \times \mathbb{T}^{\infty}$ by $\Psi \mapsto\left(\left(\left|\Psi_{j}\right|\right)_{j=1}^{\infty},\left(\arg \Psi_{j}\right)_{j=1}^{\infty}\right)$. We denote by the same symbol $U$ the map $\left(\left(\left|\Psi_{j}\right|\right)_{j=1}^{\infty},\left(\arg \Psi_{j}\right)_{j=1}^{\infty}\right) \mapsto\left(\left(\left|\Psi_{j}\right|\right)_{j=1}^{\infty},\left(\arg \Psi_{j}-h_{j}\right)_{j=1}^{\infty}\right)$. We moreover denote by $U_{\mathbb{T}}: \mathbb{T}^{\infty} \rightarrow \mathbb{T}^{\infty}$ the $\operatorname{map}\left(\theta_{j}\right)_{j=1}^{\infty} \mapsto\left(\theta_{j}-h_{j}\right)_{j=1}^{\infty}$.

The following lemma is now obvious.

Lemma 7 (Quantum Liouville's theorem). The unitary operator $U_{\mathbb{T}}$ preserves the measure $\lambda$ on $\mathbb{T}^{\infty}$.

Definition 2. We say a sequence of real numbers $\left(\theta_{j}\right)_{j=1}^{\infty}$ is rationally independent if every finite subsequence $\left(\theta_{j(1)}, \ldots, \theta_{j(n)}\right)$ satisfies $\sum_{k=1}^{n} \theta_{j(k)} m_{k} \notin \mathbb{Z}$ for all sequence of integers $\left(m_{k}\right)$.

Theorem 8. Let $\left(h_{j}\right)_{j=1}^{\infty}$ be the eigenvalues of $H$. Assume $\left(h_{j} / 2 \pi\right)_{j=1}^{\infty}$ is rationally independent. Then the operator $U_{\mathbb{T}}$ is ergodic on $\mathbb{T}^{\infty}$ with respect to $\lambda$.

Proof. We first recall that the Borel $\sigma$-algebra of $\mathbb{T}^{\infty}$ is generated by cylinder sets. To show $U_{\mathbb{T}}$ is ergodic, it is sufficient to prove that every set in a cylinder set has the ergodic property: Every finite dimensional projection of $U_{\mathbb{T}}$ is ergodic. However, from the assumption, every finite subsequence of $\left(2 \pi h_{j}\right)$ is also rationally independent and therefore it is ergodic on the finite dimensional torus (see [13, Theorem 3.2]).

\section{REFERENCES}

[1] F. Bayart and É. Matheron, Dynamics of linear operators, Cambridge university press, 2009.

[2] P. Bocchieri and A. Loinger, Quantum recurrence theorem, Phys. Rev. 107 (1957), no. 2, 337-338.

[3] Z. Brzezniak, private communication, 2010.

[4] Z. Brzeźniak and Antoni Leon Dawidowicz, On periodic solutions to the von Foerster-Lasota equation, Semigroup Forum 78 (2009), 118-137.

[5] A. L. Dawidowicz, N. Haribash, and A. Poskrobko, On the invariant measure for the quasilinear Lasota equation, Math. Meth. Appl. Sci. 30 (2007), 779-787.

[6] R. Duvenhage, Recurrence in quantum mechanics, Intern. J. Theor. Phys 41 (2002), no. 1, $45-61$.

[7] T. Hogg and B. A. Huberman, Recurrence phenomena in quantum dynamics, Phys. Rev. Letters 48 (1982), 711-714.

[8] K. Itô, Complex multiple Wiener integral, Japan J. Math. 22 (1953), 63-86.

[9] _ Wiener integral and Feynman integral, Proc. Fourth Berkeley Symp. Math. Statist. Prob. II, 1960, pp. 119-132.

[10] _ Generalized uniform complex measures in Hilbertian metric space with their application to Feynman integral, Proc. Fifth Berkeley Symp. Math. Statist. Prob. II, 1965, pp. $145-161$. 
[11] S. Kakutani, Notes on infinite product measure spaces, I, Proc. Imp. Acad. Tokyo 19 (1943), 148-151.

[12] R. Lyons, Seventy years of Rajchman measures, J. Fourier Anal. Appl. Publ. Res. Kahane Special Issue (1995), 363-377.

[13] R. Mañé, Ergodic theory and differentiable dynamics, Springer-Verlag, 1987.

[14] I. C. Percival, Almost periodicity and the quantal H theorem, J. Math. Phys. 2 (1961), 235239.

[15] C.-A. Pillet, Quantum dynamical systems, Open quantum systems. I, 2006, Lecture Notes in Math., 1882, pp. 107-182.

[16] M. Reed and B. Simon, Methods of modern mathematical physics, vol. IV: Analysis of operators, Academic Press, New York (1978).

[17] L. S. Schulman, Note on the quantum recurrence theorem, Phys. Rev. A 18 (1978), no. 5, 2379-2380.

[18] I. Shigekawa, Itô-Wiener expansions of holomorphic functions on the complex Wiener space, Stochastic Analysis, 1991, pp. 459-473.

[19] K. B. Sinha, On the absolutely and singularly continuous subspaces in scattering theory, Ann. Inst. Henri Poincaré (1977), 263-277.

[20] P. Stollmann, Caught by disorder: Bound states in random media, Birkhäuser, 2001.

[21] H. Sugita, Properties of holomorphic Wiener functions-skeleton, contraction, and local Taylor expansion, Probab. Theory Relat. Fields 100 (1994), 117-130.

Department of Mathematical Sciences, Shinshu University, Asahi 3-1-1, MatSUMOTO 390-8621, JAPAN.

E-mail address: otobe@math.shinshu-u.ac.jp

Fiber-Nanotech Young Researcher Empowerment Center, Shinshu University, AsAhi 3-1-1, Matsumoto 390-8621, JAPAN.

E-mail address: isasaki@shinshu-u.ac.jp 